\title{
Anomalous Dynamics of Forced Translocation
}

\author{
Yacov Kantor ${ }^{1, *}$ and Mehran Kardar² \\ ${ }^{1}$ School for Physics and Astronomy, Raymond and Beverly Sackler \\ Faculty of Exact Sciences, Tel Aviv University, Tel Aviv 69978, Israel \\ ${ }^{2}$ Department of Physics, Massachusetts Institute of Technology, Cambridge, Massachusetts 02139
}

(Dated: February 12, 2018)

\begin{abstract}
We consider the passage of long polymers of length $N$ through a hole in a membrane. If the process is slow, it is in principle possible to focus on the dynamics of the number of monomers $s$ on one side of the membrane, assuming that the two segments are in equilibrium. The dynamics of $s(t)$ in such a limit would be diffusive, with a mean translocation time scaling as $N^{2}$ in the absence of a force, and proportional to $N$ when a force is applied. We demonstrate that the assumption of equilibrium must break down for sufficiently long polymers (more easily when forced), and provide lower bounds for the translocation time by comparison to unimpeded motion of the polymer. These lower bounds exceed the time scales calculated on the basis of equilibrium, and point to anomalous (sub-diffusive) character of translocation dynamics. This is explicitly verified by numerical simulations of the unforced translocation of a self-avoiding polymer. Forced translocation times are shown to strongly depend on the method by which the force is applied. In particular, pulling the polymer by the end leads to much longer times than when a chemical potential difference is applied across the membrane. The bounds in these cases grow as $N^{2}$ and $N^{1+\nu}$, respectively, where $\nu$ is the exponent that relates the scaling of the radius of gyration to $N$. Our simulations demonstrate that the actual translocation times scale in the same manner as the bounds, although influenced by strong finite size effects which persist even for the longest polymers that we considered $(N=512)$.
\end{abstract}

PACS numbers: 05.40.-a 05.40.Fb 36.20.Ey 87.15.Aa

\section{INTRODUCTION}

Translocation of a polymer through a narrow pore in a membrane is important to many biological processes, such as the injection of viral DNA into a host, DNA packing into a shell during viral replication, and gene swapping through bacterial pili [1]. Translocation also has practical applications in genetics as in cell transformation by DNA electroporation [1], and in gene therapy 2]. This has inspired a number of recent in vitro experiments, including the electric field-induced migration of DNA through microfabricated channels [3] , or through an $\alpha$-hemolysin protein channel in a membrane [4, 5]. Experiments are motivated by the possibility to "read-off" a DNA or RNA sequence by tracking its passage through a pore [4, [5, [6].

Translocation of a polymer involves both molecular considerations, such as the shape of the pore channel and its interactions with DNA, as well as more macroscopic factors such as the statistics and dynamics of the long polymer. It is the universal features of the latter which are the focus of this paper. While worming its way through the hole, the segments of the polymer on each side of the membrane can "explore" many possible configurations. The number of allowed configurations actually is least when the polymer is halfway through the hole, presenting an entropic barrier. In this regard translocation resembles other entropically controlled polymer sys-

*Electronic address: kantor@post.tau.ac.il

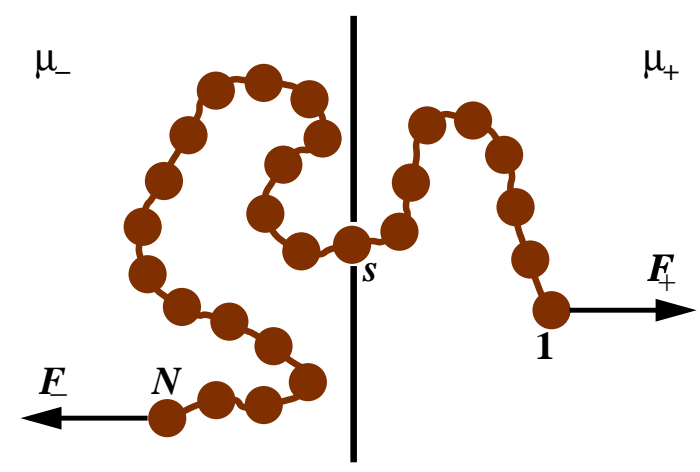

FIG. 1: Schematic depiction of polymer translocation from the left side of a membrane to its right side. (Throughout the paper we shall follow this convention for the direction of transport.) The number $s$ of the monomer at the hole is denoted the "translocation coordinate."

tems, such as polymer trapping in random environments [7, 8, 9], DNA gel electrophoresis [10] or reptation [1], where the geometry of the obstacles constrains the kinetics of the polymer.

A number of recent theoretical works have shed light on the translocation process [4, 12, 13, 14, 15, 16, 17], which is schematically depicted in Fig. 1] A single variable $s$ representing the monomer number at the pore 12, 15, 18] indicates how far the polymer has progressed, and is a natural variable for describing this problem. Due to its resemblance to the 'reaction coordinate' for chemical processes, we call $s$ the translocation coordinate. If $s$ changes very slowly, such that the polymer segments on both sides of the membrane have time to equilibrate, 
the mean force acting on the monomer in the hole can be determined from a simple calculation of free energy, and the translocation problem is then reduced to the escape of a 'particle' (the translocation coordinate) over a potential barrier. In the following, we shall refer to this limit as Brownian translocation, but shall demonstrate that the required equilibration is not tenable for long enough polymers.

In many experimental situations the polymers are not very long and the observed behavior strongly depends on the properties of the polymer and the pore. Thus introduction of some specific features into the channel properties [15, 19], or into interparticle potential [20, 21], may provide qualitative explanations of the observed behavior. While the theoretical understanding of the experiments is growing, we are still quite far from quantitative understanding of many observed features 22]. In this work, we restrict out attention to qualitative features of very long polymers that are independent of the details of the pore or inter-monomer potentials. Consequently, we restrict ourselves to simple models. Furthermore, since we are interested in contrasting the behavior of phantom polymers, in which the monomers do not interact with each other, with self-avoiding ones, in which monomers repel each other at short distances, we perform simulations in two-dimensional (2D) space, where such differences are more pronounced.

The reduction of the motion of a large molecule to a single-particle problem ignores the fact that the positions of the monomers have strong correlations 11], leading to non-trivial dynamical effects [11, 23]. In particular, in a dilute solution of polymers in a good solvent, on time scales shorter than the overall relaxation time of the polymer, the motion of monomers is characterized by anomalous dynamics [24, 25]. Not surprisingly, such effects are also present in translocation. In a previous work we demonstrated [26] that the scaling of the translocation times with the number of monomers has a power-law dependence which cannot be derived from Brownian motion of a particle over a barrier, but rather follows from general scaling considerations. In this work, we focus on consequences of anomalous dynamics in the presence of a force.

The rest of this paper is organized as follows. In Sec. II we review the limit of very slow translocation, in which case the problem can be reduced to the Brownian motion of a single coordinate. We demonstrate that the requirement of equilibration breaks down for long polymers, especially in the presence of a force pulling the polymer to one side. We also demonstrate the importance of how such a force is applied to the polymer, contrasting the cases of a polymer pulled by the end, with one forced into a favorable environment. Lower bounds on the translocation times are obtained in Sec. III by comparison with the unimpeded motion of a polymer. The long time scale for equilibration of a forced polymer is due to its change of shape, e.g. into a stretched sequence of blobs when pulled at one end. Exactly how this change of shape is achieved through transmission of the force from one end to another is explicitly shown in Appendix \$ which solves this problem for a one-dimensional (1D) phantom polymer. Our other model system, the self-avoiding two dimensional polymer is discussed in Appendix B The bounds from unimpeded motion serve a convenient reference point for discussion of anomalous processes that are described in detail in Sec. IV] In particular, we find that the actual translocation times have the same scaling behavior as the lower bounds.

\section{BROWNIAN TRANSLOCATION AND ITS LIMITATIONS}

If the translocation process is sufficiently slow, at each stage the statistics of the segments will be governed by the equilibrium Boltzmann weight. If so, the dynamics is constrained to reproduce the corresponding statistics. A simple method to achieve this is to focus on the translocation coordinate $s$, and to write down a stochastic Langevin equation for its evolution. This procedure, which we shall refer to as Brownian translocation, is the chief tool employed in most analytical studies 12, 15, 18], and shall be reviewed in this Section.

\section{A. Unforced motion}

In the absence of an external potential, the entropic contributions to the free energy of the two polymeric segments result in a free energy $\mathcal{F}(s)=\gamma k_{B} T \ln [(N-s) s]$ 12, 15, 18]. Note that there is a decrease in the number of possible states as the polymer threads the hole, which can be regarded as an entropic barrier. For phantom polymers (random walks) $\gamma$ is equal to $1 / 2$, while for self-avoiding polymers it depends on the dimensionality of space. From this free energy, we can construct a Langevin equation $\dot{s}=-m \partial \mathcal{F} / \partial s+\eta(t)$, where $m$ is a mobility coefficient indicating how easily the polymer is pulled through the hole. To obtain the correct Boltzmann statistics, the noise $\eta(t)$ has to be uncorrelated at different times, with a variance equal to $m k_{B} T$.

As demonstrated in Ref. [26], the corresponding Fokker-Planck equation for the probability $p(s, t)$ can be made independent of $N$ by a simple change of variables $s \rightarrow s N$ and $t \rightarrow t / N^{2}$. Consequently, the average translocation time $\tau$ (and its fluctuations) must scale as $N^{2}$ for any $\gamma$. In fact, a very similar distribution for the transit time is obtained by ignoring the potential barrier completely (setting $\gamma=0$ ). In this limit the translocation coordinate simply undergoes diffusion, i.e. at time scales much shorter than typical translocation times we $\operatorname{expect}\left\langle\left(s\left(t^{\prime}\right)-s(t)\right)^{2}\right\rangle \propto\left|t-t^{\prime}\right|$. 


\section{B. Pulling on the ends}

The advent of optical tweezers has made it possible to manipulate single macromolecules. A common procedure is to attach latex balls to the end(s) of a polymer (such as DNA), and then to manipulate the balls by optical tweezers [27, 28]. While this is not the method commonly used in the translocation experiments, it motivates an interesting extension of the previous calculation. Let us imagine that through an optical tweezer set-up forces $F_{+}$and $F_{-}$ are applied to the two ends of the polymer (perpendicular to the wall), as in Fig. 1]

A polymer configuration in which the ends are separated by a distance $\vec{r}$ gets an additional Boltzmann weight of $\exp \left(\vec{F} \cdot \vec{r} / k_{B} T\right)$. For a Gaussian polymer of length $N$, integration over all locations $\vec{r}$ leads to a contribution of $\exp \left[N\left(F a / k_{B} T\right)^{2}\right]$ to the partition function ( $a$ is a microscopic length scale). Restricting the integration over $\vec{r}$ only to half space (as appropriate for the translocation problem), does not change the qualitative form, i.e., in the presence of the force the free energy acquires a term proportional to $k_{B} T N\left(F a / k_{B} T\right)^{2}$.

The above argument can be generalized to a selfavoiding polymer by noting that due to dimensional considerations a force $F$ should always appear in the combination $F R / k_{B} T$, where $R$ is a characteristic size of the polymer, such as its radius of gyration $R_{g}$. Quite generally $R \sim a N^{\nu}$, with $\nu=1 / 2$ for a phantom (nonself-interacting) polymer, and $\nu=3 / 4$ and 0.59 for selfavoiding polymers in space dimensions $d=2$ and 3 , respectively [29]. The contribution of the force to the free energy can thus be written as $k_{B} T \Phi\left(F a N^{\nu} / k_{B} T\right)$. When the force is sufficiently strong to deform the polymer into a sequence of blobs (we shall argue later that this is the relevant regime for translocation), the free energy is expected to be linear in $N$, necessitating $\Phi(x) \sim x^{1 / \nu}$. Such considerations thus imply a free energy contribution of $N k_{B} T\left(F a / k_{B} T\right)^{1 / \nu}$. In the specific case of translocation, adding up the contributions from the two segments leads to

$$
\mathcal{F}(s) \sim k_{B} T\left[s\left(\frac{F_{+} a}{k_{B} T}\right)^{1 / \nu}+(N-s)\left(\frac{F_{-} a}{k_{B} T}\right)^{1 / \nu}\right] .
$$

The corresponding Langevin equation for the translocation coordinate is now

$$
\dot{s}=\lambda\left(F_{+}^{1 / \nu}-F_{-}^{1 / \nu}\right)+\eta(t)
$$

where we have absorbed various coefficients into the parameter $\lambda$. Note that the average velocity has a nonlinear dependence on the forces; in the case of $F_{-}=0$ growing as $F_{+}^{1 / \nu}$. Consequently, the translocation time in such a set up should decrease with the applied force as $\tau \propto N / F_{+}^{1 / \nu}$. Note that this expression breaks down for small forces, where the typical translocation time is controlled by the diffusive fluctuations. The distinc- tion between weak and strong force regimes has a specific meaning in the context of polymers, and quantified through the scaling combination $\tilde{f} \equiv \mathrm{FaN}^{\nu} / k_{B} T$. For weak forces this combination is small, and the equilibrium polymer shape is not changed. For strong forces $\tilde{f} \gg 1$, and the polymer becomes stretched. The same division applies to forces that are strong enough to overcome the diffusive character of the translocation coordinate.

\section{Chemical potential difference}

A more common situation for translocation is when the environments separated by the membrane are not equivalent, so that the polymer encounters a chemical potential difference between the two sides. In this case, the leading contribution to the free energy is $\mathcal{F}(s)=$ $\mu_{+} s+(N-s) \mu_{-}$, and the Langevin equation takes the form

$$
\dot{s}=\lambda^{\prime} \Delta \mu+\eta(t)
$$

with $\Delta \mu \equiv\left(\mu_{-}-\mu_{+}\right)>0$. In this case the average translocation velocity is predicted to be proportional to $\Delta \mu$, leading to typical exit times that scale as $N / \Delta \mu$ for large $\Delta \mu$. It is possible to envision situations in which the polymer is forced (or hindered) by a combination of both a chemical potential difference, and forces applied to the two ends. For the corresponding Langevin equation, we merely need to add the force contributions in Eqs. (2) and (3).

In the experiments of Meller et al. [5], translocation is driven by an electric potential difference between the two sides of an artificial membrane suspended in a liquid. It is commonly assumed that since the conductivity of the liquid is significantly higher than the conductivity of the membrane, the liquid on each side of the membrane is an equipotential. The voltage drop then occurs only across the membrane, and is experienced only by the (charged) monomers moving through the pore 22]. If so, the voltage difference is equivalent to the chemical potential difference discussed above. However, the previous results with force acting only on the end monomers serve as a warning that the results are quite sensitive to where the force is applied to the polymer. It would thus be reassuring to carry out a more precise calculation of the electric field in the vicinity of the pore, and how it acts upon the monomers.

\section{Limitations}

The analytical procedure outlined in this section rests on the assumption that the two polymeric segments have come to equilibrium, so that the corresponding free energy can be used to construct a Langevin equation. The minimal requirement is that the typical translocation 
time $\tau$ should exceed the equilibration time $\tau_{\text {equil }}$ of a polymer. For a chain of finite size, it is always possible to achieve this by designing the pore to have a large friction coefficient. In Ref. [15], it is argued that this is the case applicable to the experiments of Ref. [5]. However, the equilibration time of a polymer depends strongly on its length, scaling as $\tau_{\text {equil }}(N) \propto N^{z \nu}$. As discussed in the next section, the exponent $z \nu$ is typically larger than 2 for Brownian dynamics of self-avoiding polymers (and equal to 2 for a phantom polymer).

Since typical transit times for unforced translocation scale as $N^{2}$, it is possible to imagine that the formalism may apply to phantom polymers. Indeed there is some evidence of this from numerical simulations [30], although with an inexplicably large friction coefficient. The situation becomes worse in the presence of a force (either imposed by pulling or a chemical potential difference), in which case typical translocation times are predicted to be proportional to $N$. In the latter case, the range of applicability of 'Brownian translocation' is even further limited.

\section{UNIMPEDED MOTION OF A POLYMER}

Since the collective dynamics of the passage of polymer through the pore is hard to treat analytically, as a first step we shall derive lower bounds on the characteristic time scale. The key observation is that the restriction that the monomers should sequentially pass through a hole in a membrane can only impede the motion of the polymer. Hence the time scale for the polymer to travel the same distance in the absence of the wall should be a generous lower bound to its translocation time. In this Section we shall thus explore the time scale for unimpeded motion of the polymer in the circumstances of interest.

\section{A. Unforced diffusion}

In the unforced limit, the translocating polymer simply goes from one side of the membrane to the other by 'diffusion.' In the process, the center of mass of the polymer moves a distance of the order of a typical size, say the gyration radius of $R_{g}$. How long does it take for a polymer to move a similar distance without the constraints imposed by the pore and the wall? In the absence of hydrodynamic interactions, the diffusion constant $D$ of a polymer is related to the diffusion constant $D_{0}$ of a single monomer by $D=D_{0} / N$. Consequently, the time that a polymer needs to diffuse its own radius of gyration scales as $N^{1+2 \nu}$ 11]. (This is also the relaxation time of the slowest internal mode of a polymer [11], and is called the Rouse equilibration time.) For self-avoiding polymers $\nu>1 / 2$, and the equilibration time is clearly longer than that obtained for translocation though a hole in the wall assuming Brownian translocation. The Rouse time scale of $N^{1+2 \nu}$ should thus be a lower bound to the correct translocation time.

\section{B. Pulling on the end}

Now consider a polymer that is being pulled by one end. The regime of interest to us is when the force is strong enough to deform the shape of the polymer. The equilibrium shape of the polymer is then a stretched sequence of 'blobs' 11]. The number of monomers per blob $N_{B}$ is such that force acting on it is marginally strong, and obtained from $F a N_{B}^{\nu} \sim k_{B} T$. The typical size of each blob is thus $R_{B} \sim a N_{B}^{\nu} \sim k_{B} T / F$, while the number of blobs is $N / N_{B} \sim N\left(F a / k_{B} T\right)^{1 / \nu}$. The overall length of the stretched chain is now $R(F) \sim R_{B}\left(N / N_{B}\right) \sim$ $a N\left(F a / k_{B} T\right)^{1 / \nu-1}$. The mobility of the center of the mass of a polymer of length $N$ is proportional to $1 / N$, and since there is only a force applied to one monomer, its net velocity scales as $F / N$. The characteristic relaxation time is the same as the time scale of the polymer moving a distance of order of its size, and hence behaves as

$$
\tau_{\text {equil }}(F) \sim \frac{R(F)}{v(F)} \propto N^{2} F^{-2+1 / \nu} .
$$

(The same conclusion is obtained if we start with a globular polymer and then apply the force to one end, and wait until the other end feels the force.)

Note that upon approaching the boundary between weak and strong forces at $F \propto N^{-\nu}$, we regain the equilibration time $N^{1+2 \nu}$ for unforced polymers. However, the result in Eq. (4) is only valid for $N^{-\nu} \leq \mathrm{Fa} / k_{B} T \leq 1$, since for stronger forces, as we shall see in the specific models described in the next Section, the velocity of the monomer (and hence of the entire chain) saturates.

Unimpeded motion of the pulled polymer thus places a lower bound of $N^{2}$ on the translocation time, far exceeding the time scale $(\propto N)$ calculated in the previous section. It may not be readily apparent how the force applied to one end of the polymer is transmitted to the other end, and why the qualitative picture of blobs presented above is valid. There is actually one limit in which the problem of pulling a polymer by the end can be solved analytically, and that is for a $1 \mathrm{D}$ phantom polymer. In Appendix $\mathrm{A}$ this model and the corresponding analysis are presented in some detail.

\section{Mimicking a chemical potential difference}

It is difficult to come up with an unhindered situation that best resembles the case of a chemical potential difference across a membrane. Absent the restrictions imposed by the membrane, there is now a force that is applied to a single monomer, at the spatial position where the membrane would reside. Unlike the previous case, the 
monomer to which the force is applied now changes constantly. There is thus no incentive for a drastic change in the shape of polymer, and we assume that the scaling of the size remains the same, i.e. $R \sim a N^{\nu}$, independent of $\Delta \mu$. At each moment there is a force of $\Delta \mu / a$ applied to the entire polymer, as a consequence of which its center of mass should move with a velocity $v \propto \Delta \mu / N$. We thus conclude that the time for such unhindered motion scales as

$$
\tau(\Delta \mu) \sim \frac{R}{v} \propto \frac{N^{1+\nu}}{\Delta \mu} .
$$

Note that to recover the equilibration time of the unforced polymer we have to set $\Delta \mu \propto N^{-\nu}$ in the above equation. While this is the same scaling form as that of a force applied to the end, it is different from the weak/strong criterion that would have been deduced on the basis of energetics $\left(\Delta \mu N \sim k_{B} T\right)$. This is a reflection of the manner in which we introduced the unimpeded version (as a force, rather than a chemical potential difference.) Nonetheless, we still expect the velocity, and hence the time scale in Eq. (5) to saturate for $\Delta \mu \sim k_{B} T$, as explicitly demonstrated for the models considered in the next Section.

\section{ANOMALOUS TRANSLOCATION}

Having established some some (presumably generous) lower bounds, we now would like to focus on the true asymptotic dynamics of translocation. Given the limitations of analytical studies, the chief tool employed in this section is numerical simulations. Interestingly, we find that the lower bounds obtained in the previous section are actually quite restrictive.

\section{A. Sub-diffusive behavior of unforced motion}

In a previous work [26], we made a detailed study of the $N$-dependence of the mean translocation time. One of the central conclusions was that in the case of Brownian dynamics of a self-avoiding polymer, the translocation time scales as

$$
\tau \sim N^{1+2 \nu} .
$$

This is of the same order as the equilibration time of a polymer of length $N$, and also demonstrates that the actual exit time scales in the same manner as the bound established in the previous section.

The above $N$-dependence of $\tau$ is inconsistent with simple diffusion of the translocation coordinate $s(t)$, reflecting the constraints imposed by the collective motion of the entire polymer. A related situation occurs for the fluctuations of a labeled monomer in space, which are also anomalous and sub-diffusive 24 on time scales shorter than the equilibration time. Following this analogy, we

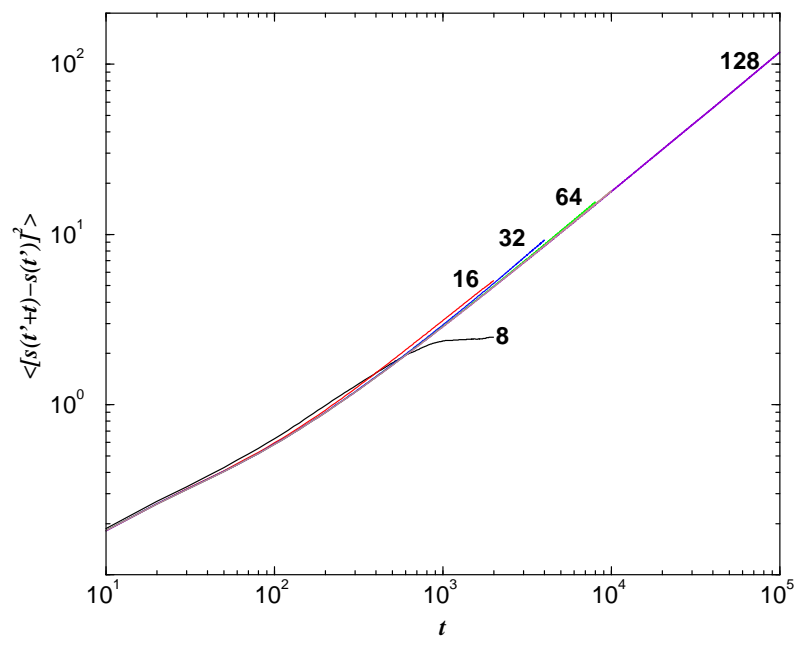

FIG. 2: Temporal fluctuations of the monomer number $s$ located at the hole, averaged over the initial time $t^{\prime}$ and over 1000 independent simulations, for polymers of lengths $N=8$, $16,32,64$, and 128 .

suggested [26] that the short time fluctuations of $s(t)$ follow the anomalous diffusion relation

$$
\left\langle\Delta s^{2}(t)\right\rangle \sim t^{2 \zeta}
$$

For Eqs. (6) and (7) to be consistent, we must obtain $\Delta s$ of order $N$, when $t$ of order $\tau$, leading to the exponent relation $\zeta=1 /(1+2 \nu)$. Note that for a phantom polymer $\nu=\zeta=1 / 2$, i.e. the anomaly disappears in this limit, and the process becomes diffusive. (This differs from the corresponding motion of a labeled monomer [24, 25], which remains anomalous even for a phantom polymer.) This is consistent with a detailed study of a three-dimensional phantom polymer by Chern et al. 30] which concluded that the results may be interpreted in terms of diffusive motion of the translocation coordinate over a barrier. Such correspondence is likely a fortuitous coincidence for phantom polymers, and even in this case, the value of the effective diffusion constant could not be obtained from the geometrical features of the model [30].

To establish the anomalous nature of (unforced) translocation dynamics, we carried out Monte Carlo (MC) simulations on a model of two-dimensional selfavoiding polymers, described in Appendix B Simulations in two (rather than three) dimensions have the advantage of relative ease, and stronger differences from phantom polymers. We followed the dynamics of $s(t)$, focusing on the quantity $\Delta s^{2} \equiv\left(s\left(t^{\prime}+t\right)-s\left(t^{\prime}\right)\right)^{2}$. This correlation function is depicted in Fig. 2, and was obtained by averaging over $t^{\prime}$ of over 1000 independent simulations for $N=8,16,32,64$ and 128 . Two cautionary points must be made in considering this data: The first is that we have no a priori assurance that this process is stationary; the results may depend on both $t$ and $t^{\prime}$, and consequently influenced by the averaging over $t^{\prime}$. (For example, choosing a short averaging range may increase 


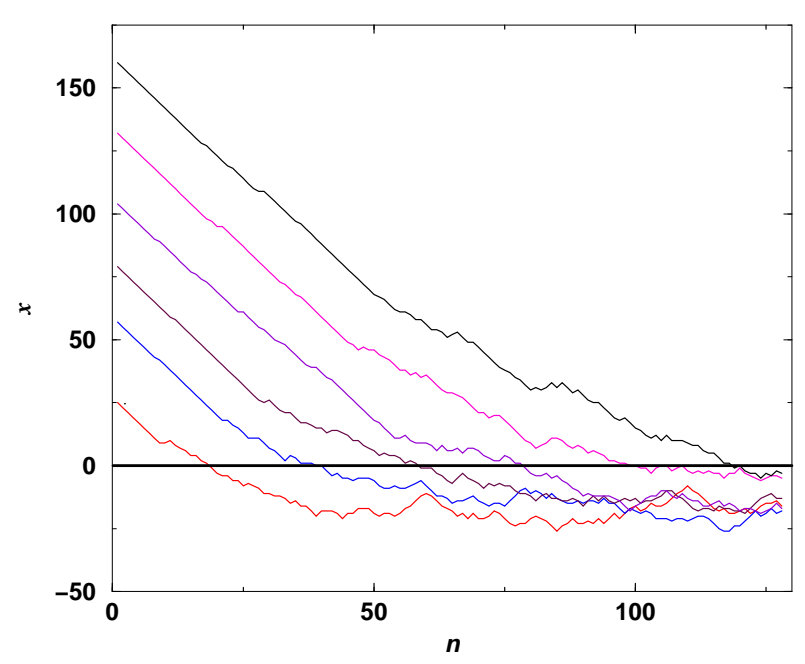

FIG. 3: "Snapshots" of a 128-monomer phantom polymer passing through a membrane in one dimension. Each line depicts the position $x$ (in lattice units) of the $n$th monomer at a fixed time. Different lines correspond to times when the 20th, 40th, ... 120th (bottom-left to top-right) monomer crosses the membrane (the thick line at $x=0$ ).

the effect of the initial conditions. We performed averaging over shorter ranges of $t^{\prime}$ and did not see significant differences in the correlation functions.) Secondly, for each value of $t$, we can only include processes whose translocation time exceeds $t$; consequently, the size of the ensemble decreases with increasing $t$. (However, this effect is insignificant for $t$ several times shorter than the mean translocation time.) In Fig. 2 all available $t^{\prime}$ are included, i.e. for each polymer the statistics was collected up to the moment that the translocation was completed.

Since the values of $s$ cannot exceed $N$, as the timedifference $t$ becomes of order of $\tau, \Delta s^{2}$ saturates, as is apparent in the case of $N=8$ in Fig. 2 However, for times shorter than $\tau(N)$, the results for different lengths seem to form a single curve. This instills confidence in the quasi-stationary character of translocation on time scales shorter than $\tau$. On the logarithmic scale the graph seems to have curvature for $t<1000$. This is probably a consequence of discreteness of the model, since corresponding differences in $s$ are smaller than 5. For larger times the slope of the curve approaches 0.80 , which clearly indicates the presence of anomalous diffusion, and is consistent with the expected value $2 /(1+2 \nu)$, with $\nu=3 / 4$ for $2 \mathrm{D}$ self-avoiding walks [29]. Thus, despite its finite duration, the translocation process at short scales resembles a stationary process (at least $\Delta s^{2}$ is insensitive to $\left.t^{\prime}\right)$ which exhibits anomalous dynamics.

\section{B. Pulling on the end}

As discussed in Sec. II the polymer pulled by a force $F_{+}$is relatively undistorted as long as $\tilde{f} \equiv$

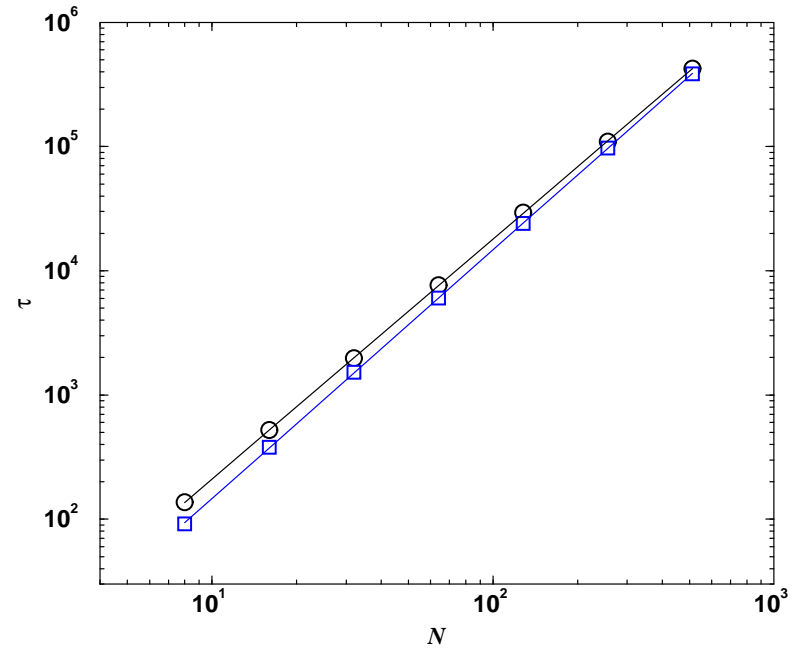

FIG. 4: Logarithmic plot of the mean translocation time as a function of length for a one-dimensional phantom polymer with an infinite force applied to one end. The circles represent passage through an opening, while the squares represent motion in the absence of a membrane. Each data point represents an average over 1000 processes.

$F_{+} a N^{\nu} / k_{B} T \ll 1$, and the corresponding translocation times are not very different to those in the absence of force. Increasing polymer length at fixed $F_{+}$ultimately leads to a regime with $\tilde{f} \gg 1$, in which the polymer is expected to be stretched into a sequence of blobs. It is the latter regime which is of interest us, and which shall be explored by examining the one- and two-dimensional polymer models introduced earlier.

\section{One-dimensional phantom polymer}

Simulations are carried out with the model 1D phantom polymer presented in Appendix $\mathrm{A}$ starting with a polymer that is equilibrated on one (say, left) side of the "membrane" (a point on a 1D lattice) with one end point held at the membrane. The "narrow opening" is implemented by allowing only sequential passage of the monomers across the membrane, i.e. the $n$th monomer can move from the left to the right only if $(n-1)$ th monomer is already on the right side. Conversely, the $n$th monomer may diffuse from the right side to the left, only if the $(n+1)$ th monomer is on the left side. The first monomer of the chain is restricted to remain on the right throughout the process. We study the dynamics of translocation as a function of the force $F$ applied to the first monomer. However, the results become independent of $F$ when the reduced force $f \equiv F a / k_{B} T$ exceeds unity, since it becomes very unlikely for the first monomer to move backwards.

Figure 3 depicts a sequence of "snapshots" of a 128monomer polymer going across the membrane. Since the maximal separation between adjacent monomers is two 
lattice spacings, the slopes of the curves are limited by 2 . Note that for $x>0$ the polymer is almost maximally stretched, while the $x<0$ configurations resemble the initial random walk state. There is also much similarity between the profile of the polymer for $x>0$, and the the steady-state configurations of a polymer moving in the absence of the membrane, as depicted in Fig. 14 of Appendix A

The results of averaging the translocation time (over 1000 realizations) are indicated by the circles in Fig. [4 The points appear to fall on a straight line in this logarithmic plot, with the slope of $1.93 \pm 0.01$ from a leastsquares-fit. There is a slight upwards curvature, and the effective slope varies from 1.84 for points with $N \leq 128$ to 1.93 for all the points, indicating potential crossover effects persisting even for $N=512$. From this data by itself it is difficult to determine the ultimate slope. However, we can compare the results with the times required to cross an imaginary membrane (i.e. unimpeded diffusion). This lower bound which was described in the previous section (and discussed in detail in Appendix (A), leads to the mean passage times depicted by the squares in Fig. [4 The extrapolated slope for this unimpeded motion is indeed 2.00. Since the unimpeded crossing times are indeed shorter, the asymptotic exponent in the presence of the membrane has to be larger than or equal to 2. (Otherwise, for sufficiently large $N$ the curves will intersect causing longer times for passage if the membrane is absent.) We therefore conclude that the translocation of a phantom polymer in 1D should asymptotically scale as $N^{2}$, saturating the bound obtained previously.

\section{Two-dimensional self-avoiding polymer}

We next study the translocation of a self-avoiding polymer in $2 \mathrm{D}$ as a function of the force $F$ applied to the first monomer. Figure 15 depicts the distribution of translocation times for a 128-monomer polymer at three values of $f=F a / k_{B} T$. As $f$ increases from 0.25 to $\infty$, the mean translocation time drops by less than one order of magnitude, and the relative width of the distribution decreases somewhat. Note that once $f \gg 1$, the first monomer always moves in the forward direction, and the results become independent of $f$.

Figure [6] summarizes the results obtained for polymer lengths $N$ ranging from 8 to 128, and for a variety of forces. Each point corresponds to an average over 1000 realizations. The figure depicts the scaled inverse translocation time as a function of the dimensionless force $f=F a / k_{B} T$. The vertical scale has been multiplied by $N^{1.87}$ to produce moderate collapse of data for different $N$ s, although as explained further on, we do not believe this to be the correct scaling factor in the asymptotic regime. As expected, the curves saturate when $f$ significantly exceeds unity.

Note that all the points in Fig. 6 belong to the regime where $\tilde{f} \gg 1$, i.e. when the shape of the polymer is ex-

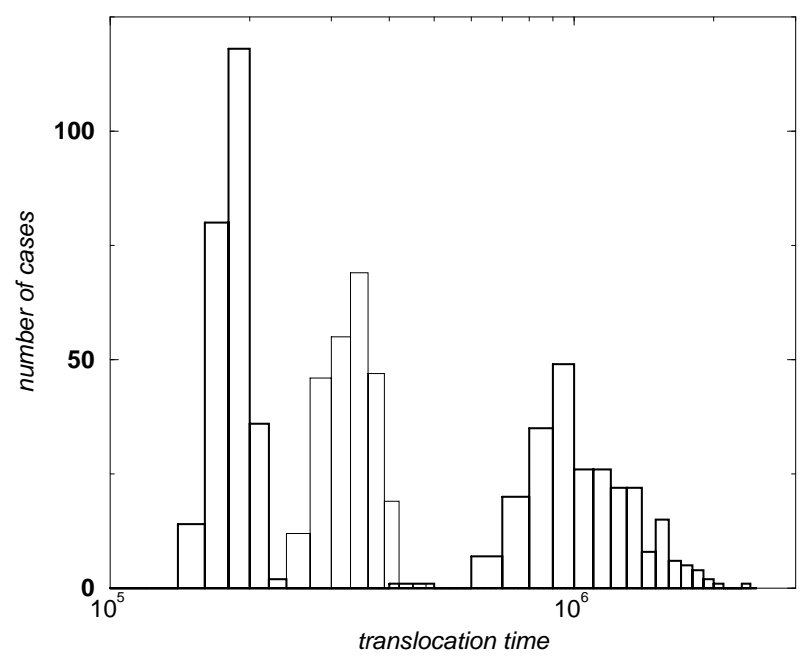

FIG. 5: The distribution of translocation times for a $2 \mathrm{D}$ polymer with $N=128$. Each histogram represents results from 250 independent translocations for forces $F a / k_{B} T=$ $0.25,1$, and $\infty$ (from right to left) applied to one end. (The horizontal axis is logarithmic.)

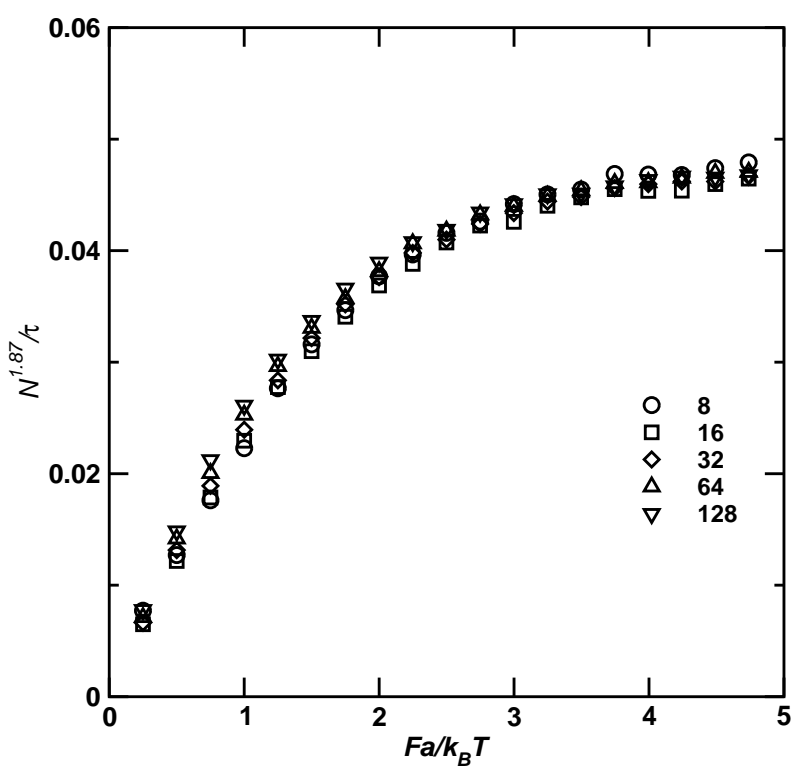

FIG. 6: Scaled inverse translocation time as a function of the reduced force $\mathrm{Fa} / k_{B} \mathrm{~T}$ applied to the end-monomer, for $N=8,16, \ldots 128$.

pected to be different from equilibrium, and stretched. This is confirmed in Fig. [7 which depicts configurations of the polymer in the process of translocation under the action of an infinite force. The front end of the polymer is quite stretched, somewhat resembling a directed random walk, suggesting that self-avoiding interactions play a secondary role in this limit. If the front part of the polymer controls the translocation time, it should have 


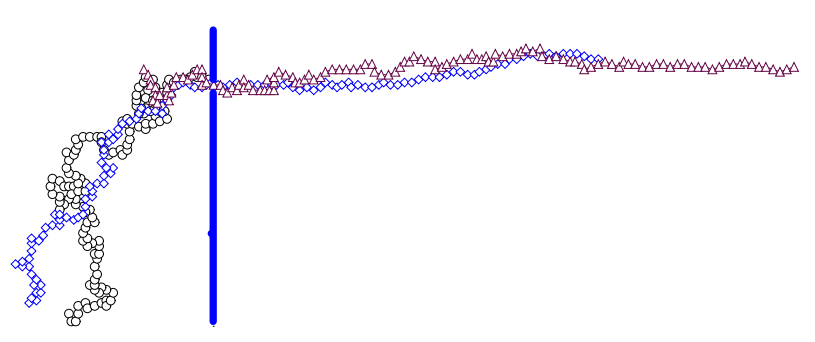

FIG. 7: Configurations of a polymer of length $N=128$, pulled through a hole by an infinite force applied to its first monomer. The circles, diamonds and triangles represent the initial configuration, and at times $t=60,000$ and 120,000 Monte Carlo time steps.

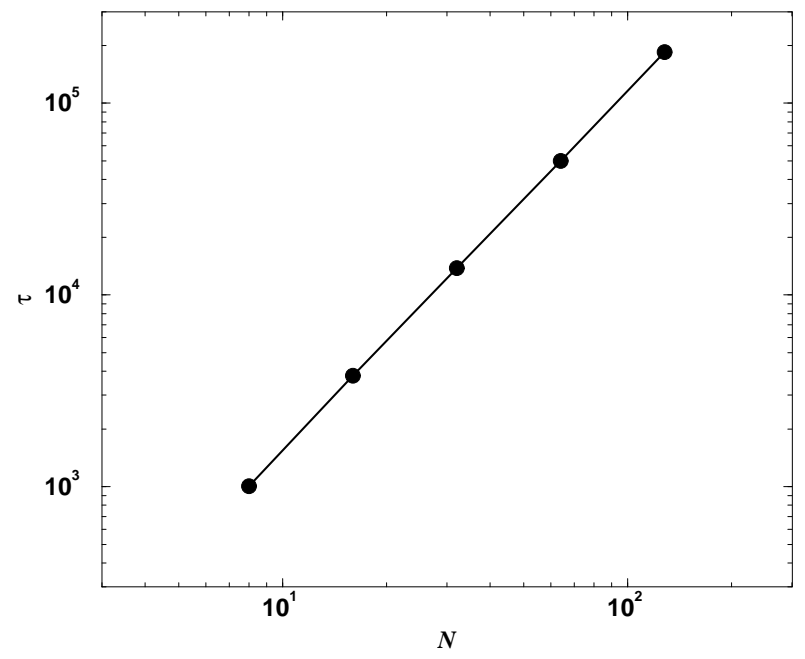

FIG. 8: Logarithmic plot of the dependence of the translocation time $\tau$ on polymer length $N$, when an infinite force is applied to the end-monomer. The line is a fit to a power law dependence with exponent 1.875 .

the same scaling with $N$ as the corresponding time for a phantom polymer, i.e. we expect $\tau \propto N^{2}$. Figure 8 depicts the dependence of $\tau$ on $N$; the effective exponent in this range is $1.875 \pm 0.005$. Although smaller than two, it is close to the value of the effective exponent of a phantom polymer in this range of lengths $N$. Given the bound presented earlier, it is reasonable to expect asymptotic convergence to this value. However, as far as we can judge by analogy to phantom polymers, we need $N$ to be much larger than 1000 to see an exponent of 2 .

\section{Chemical potential difference}

When the environments on the two sides of the membrane are different, the monomer at the pore experiences a force pushing it to the more favorable side. As explained previously, this form of forcing leads to yet a different form of asymptotic behavior which is once more explored using our two numerical models.

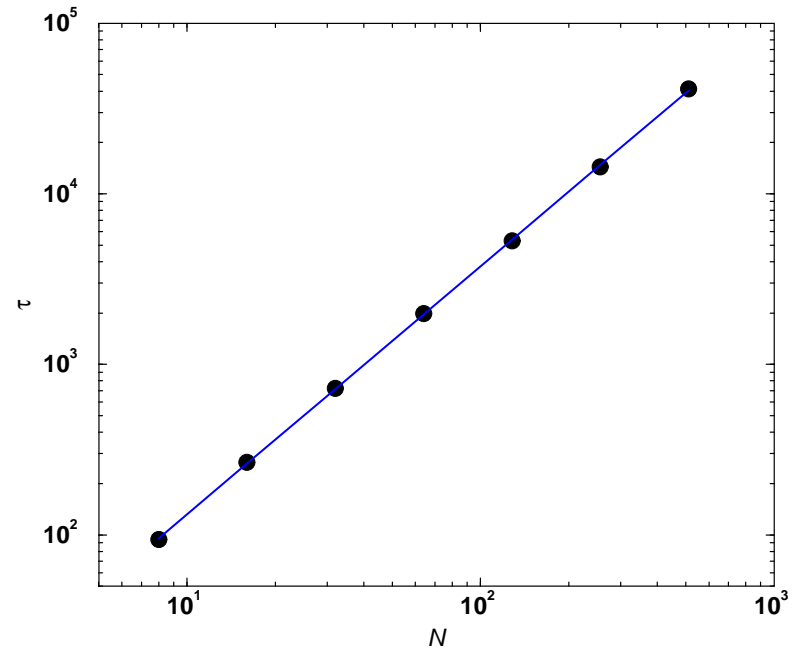

FIG. 9: Logarithmic plot of the mean translocation time as a function of polymer length for a one-dimensional phantom polymer subject to an infinite chemical potential difference. The solid line is a fit to a power law with exponent 1.45 .

\section{One-dimensional phantom polymer}

Figure 9 depicts the $N$-dependence of the translocation time $\tau$ for a 1D phantom polymer under the influence of an infinite potential difference, i.e. when the monomer at the pore can only move to one side. The data on the logarithmic plot are fitted to a straight line with exponent $1.45 \pm 0.01$, although there is a slight upward curvature even for $N=512$. Note that in the limit of large chemical potential difference, the monomers that have already crossed to the right side no longer play any role in the translocation process, which is thus constrained by the dynamics of the monomers remaining on the left side. In Sec. III we argued that the translocation time of an unimpeded phantom polymer should scale as $N^{3 / 2}$, since the leftmost monomer must travel a distance of order $N^{1 / 2}$ with a velocity of order $1 / N$. Thus $3 / 2$ should be a lower bound for the exponent characterizing the scaling of the translocation time with $N$. By comparing this limit with our numerical results, we conclude $3 / 2$ to be the true asymptotic form describing our simulations.

\section{Two-dimensional self-avoiding polymer}

Figure 10 presents the distribution of translocation times for a polymer with $N=64$ at several values of the dimensionless chemical potential difference $\Delta \mu / k_{B} T$. These distributions are quite wide - although they become (relatively) narrower with increasing $\Delta \mu$, the width of the distribution is of the same order as the average even in the limit of an infinite $\Delta \mu$. As expected, the average translocation times decrease and saturate when $\Delta \mu / k_{B} T$ exceeds unity. The results for different values of $\Delta \mu / k_{B} T$ and $N$ can be approximately collapsed by scaling $\tau$ with 


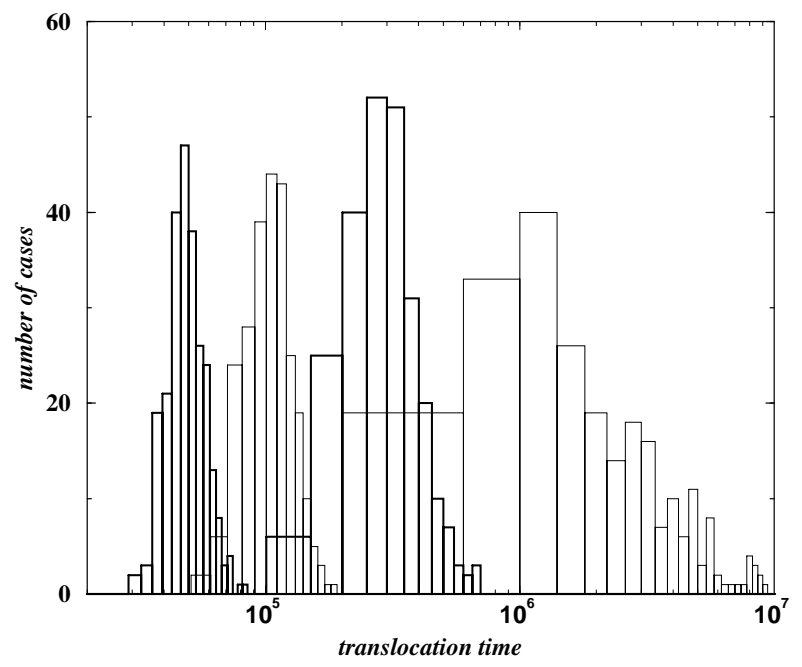

FIG. 10: Distribution of translocation times of a 64monomer polymer subject to chemical potential differences of $\Delta \mu / k_{B} T=0,0.25,0.75$, and 2 (right to left). (The horizontal axis is logarithmic.)

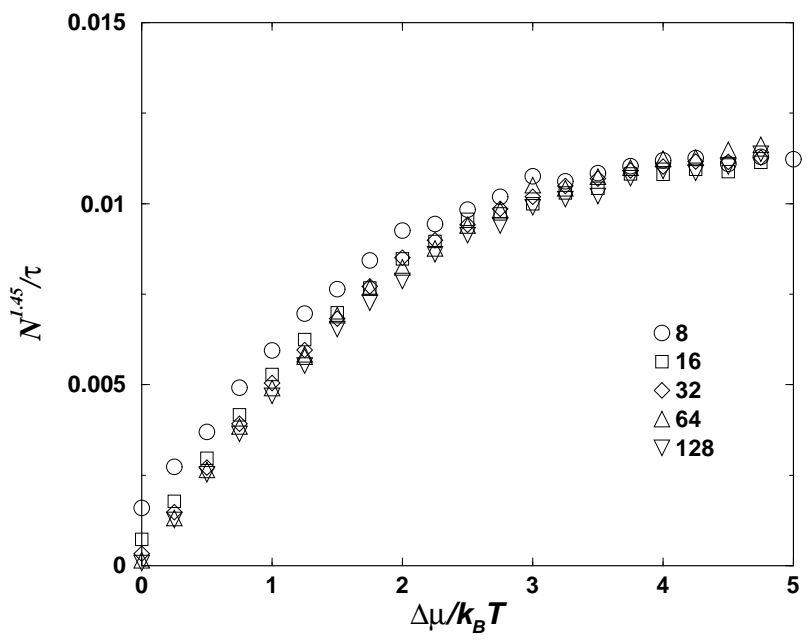

FIG. 11: Scaled inverse mean translocation time as a function of reduced chemical potential difference $\Delta \mu / k_{B} T$, for $N=8$, 16, 32, 64 and 128 .

$N^{1.45}$, as shown in Fig. 11 (where each point represents an average over 1000 independent runs). The quality of the collapse is very poor, and we shall argue that $N^{1.45}$ is not the expected asymptotic power.

Since (with the exception of $\Delta \mu=0$ ), the points in Fig. 11 correspond to a strong force at the pore, we expect the configurations of the translocating polymer to be different from those of a polymer in equilibrium. To explore this difference, in Fig. 12 we show a pair of configurations for an infinite chemical potential difference. We see that fast translocation results in a higher density of monomers immediately to the right of the pore, which may in principal slow down the process. (Recall that in the case of phantom polymers the monomers that

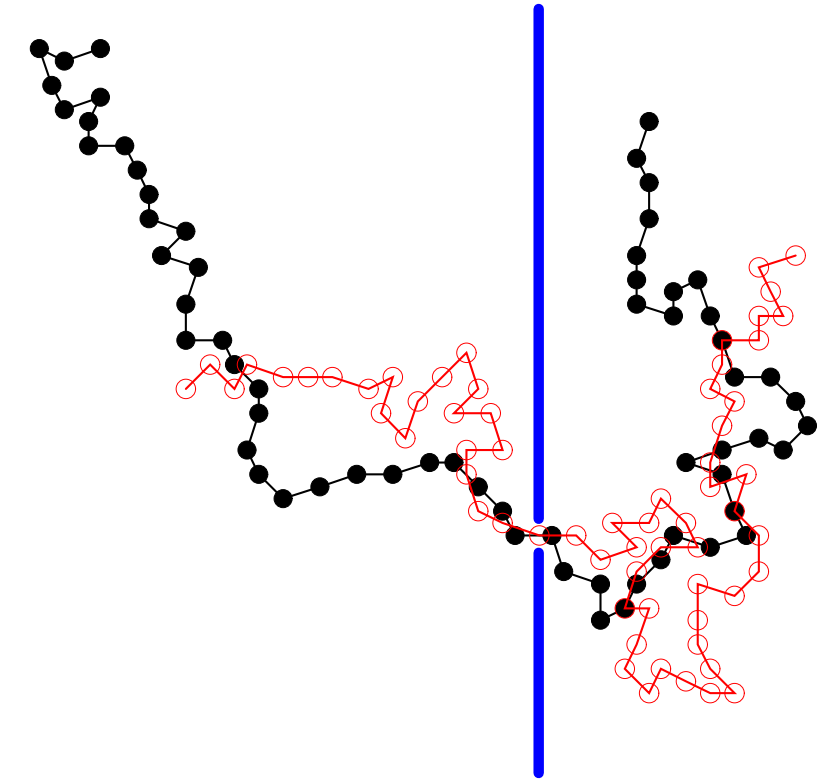

FIG. 12: Configurations of a polymer of length $N=64$ crossing a membrane from left to right under an infinite chemical potential difference. Full and open symbols represent times of $t=10,000$ and 25,000 Monte Carlo steps, respectively

have passed through the hole have no further influence.) Nevertheless, the whole process should still be bounded by the corresponding time for passage of an unimpeded polymer, as discussed in Sec. III. For considerations of this bound, the relevant time corresponded to motion of the leftmost monomer over a distance of size $R \propto N^{\nu}$, leading to a time scale growing as $N$ to a power of $1+\nu$ which in $2 \mathrm{D}$ is 1.75 .

The optimal data collapse of the inverse translocation times for $N \leq 128$ leads to an exponent 1.45 (with a rather poor data collapse). Therefore, we extended our simulations for the case of the infinite $\Delta \mu$ to larger values of $N$. Fig. 13 represents the dependence of mean translocation time on $N$, for polymer lengths up to 512. Data points with $N \leq 128$ correspond to averages over 1000 independent simulations, while $N=256$ and 512 include 300 and 130 runs, respectively. The effective slope of the fit for data points below $N=128$ is $1.45 \pm 0.01$, while all the data points produce an effective slope of $1.53 \pm 0.01$. Also by directly measuring the effective slopes between successive pairs of points we definitely see an increase, with the last pair of points giving a slope of $1.60 \pm 0.03$. However, the increase is very slow, and the uncertainties are too large to enable a reliable extrapolation to large $N$. By comparing the results to data obtained in the simulations of phantom polymers we believe that eventually the exponent will reach 1.75 ; however, this will probably happen only for $N$ significantly larger that 1000 . 


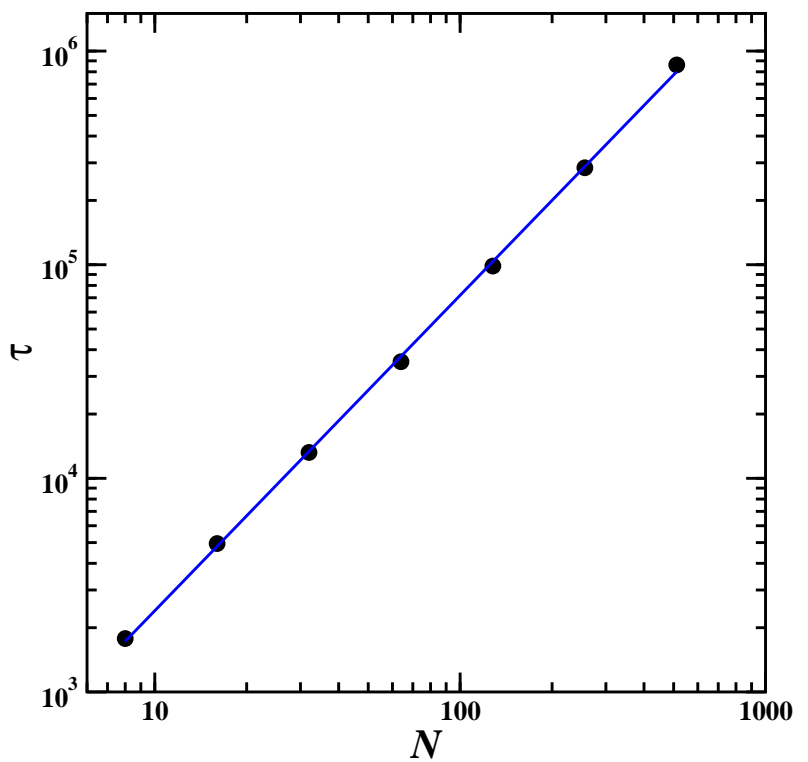

FIG. 13: Logarithmic plot of the dependence of the translocation time $\tau$ on polymer length $N$, for an infinite chemical potential difference. The solid line is a fit to a power law dependence with exponent 1.53 .

\section{DISCUSSION}

Translocation of a polymer through a pore is intrinsically a many body problem involving collective and cooperative motion of monomers crossing a membrane. If the process is sufficiently slow, it is possible for the segments on the two sides to come to equilibrium, in which case the dynamics of the translocation coordinate (the number of monomers on one side) is similar to Brownian motion of a single particle. However, the assumption of equilibrium must necessarily break down for long enough polymers; even more drastically when the polymer is pulled to one side by a force. We argued previously [26] that the collective motion of the whole polymer slows down the translocation to the extent that the corresponding dynamics is anomalous and sub-diffusive. This is explicitly verified in this paper by quantifying the temporal correlations of the fluctuating translocation coordinate. We note that there is a general theoretical framework 31] for anomalous dynamics of a single variable, which may be profitably applied to this problem.

One of the objectives of this paper was to find how dynamical anomalies affect the motion of the polymer under the action of a force. With this in mind, we also emphasized that the method by which the polymer is forced is quite important. In particular, pulling the polymer by one end leads to stretched configurations, and slower overall dynamics, compared to applying a chemical potential difference (which can modify the densities on the two sides). While pulling the polymer by optical tweezers is not the currently favored method for artificial translocation of biopolymers, for potential applications such as decoding the sequence, it should offer a better controlled procedure (whether by itself or in conjunction with a voltage difference).

To understand the time scales involved in forced translocation, we initially provided what at first glance appear to be a quite loose lower bounds by analogy to unimpeded motion of a polymer, i.e. neglecting the constraints imposed by passage of the polymer through a hole in a wall. We then performed numerical simulations on two model systems: a 1D phantom polymer, and a self-avoiding polymer in 2D. Direct interpretation of the numerical results was made difficult by very large crossover effects which persist in the length scales of 100-1000 monomers accessible to numerical study. Nonetheless, by appealing to the lower bounds found earlier, we concluded that (rather surprisingly) the actual translocation times scale in the same way as in the limit of unimpeded motion. Thus the constraints from the collective motion of the whole polymer turn out to be at least as important as those imposed by the requirement of passage through a hole.

The experiments of Ref. [5] suggest that in the range of 10 to 100 base pairs, the pulling velocity of singlestranded DNA through a nanopore is independent of $N$, but with a non-linear dependence on the applied force. In Ref. [26], we briefly speculated whether such behavior may be consistent with anomalies associated with polymeric constraints. As demonstrated in this paper, such constraints result in time scales (and hence pulling velocities) which must depend on $N$ for large enough $N$. The only case where we observe a non-linear force-velocity relation which is independent of $N$ is when short (hence equilibrated) polymers are pulled by a force applied to one end.

We hope that these results encourage further experimental and analytical studies of forced translocation. In particular, it would be interesting to better characterize the manner in which external forces act on the polymer, even in the case of a voltage difference across the membrane. Hydrodynamic effects, not considered in this paper, are also likely to play an important role. Some of these effects can be included in more realistic numerical simulations, although in that case one should keep in mind the rather long crossover times that appear to be intrinsic to this process.

\section{Acknowledgments}

This work was supported by the Israel Science Foundation grant No. 38/02 (Y.K.), and by the National Science Foundation through grant No. DMR-01-18213 (M.K.). 


\section{APPENDIX A: THE ONE-DIMENSIONAL PHANTOM POLYMER MODEL}

Self-avoiding interactions are integral to understanding the statics and dynamics of real polymers. Nevertheless, it is useful to study one-dimensional phantom polymers, with no interactions between monomers which are not adjacent along the chain. Independently of the details of the interactions of adjacent monomers, long phantom polymers are harmonic 11], in the sense that the probability distribution of the distance $\left|\mathbf{r}_{i}-\mathbf{r}_{j}\right|$ between monomers $i$ and $j$, approaches a Gaussian for large $|i-j|$. In practice, such behavior already appears for $|i-j| \approx 10$, and on such a "coarse-grained" level one can view the polymer as consisting of monomers connected by springs whose energy is proportional to $\left(\mathbf{r}_{i}-\mathbf{r}_{j}\right)^{2}$. In this limit, certain aspects of phantom polymer dynamics can be analyzed analytically, and we can compare the expected asymptotic behavior with the numerically observed dynamics. Such treatment provides both a better view of crossover effects, and produces some insights into the dynamics of more realistic (self-avoiding) models.

We employ a phantom polymer model in which the monomers are restricted to sites of a 1D lattice with spacing $a$. The polymer connectivity is implemented by requiring the distance between adjacent monomers not to exceed two lattice constants. An elementary Monte Carlo (MC) step consists of randomly picking a monomer and attempting to move it in a randomly selected direction. If an external force $F$ is applied to the first monomer of the chain, then the probability to make a step in the direction opposite to the force is proportional to $\exp \left(-a F / k_{B} T\right)$. A MC time unit corresponds to $N$ attempts to move monomers.

We first performed simulations of polymer motion when $F \rightarrow \infty$, in the absence of a membrane. Figure 14 depicts several examples of spatial configurations in steady state, i.e. when the initial spatial configuration has been forgotten. The resulting profiles can be explained analytically by noting that in the Gaussian limit the equation of motion of a $1 \mathrm{D}$ phantom polymer is given by

$$
\frac{\partial^{2} x}{\partial n^{2}}=\frac{\partial x}{\partial t},
$$

where we have used dimensionless units, (distance in lattice constants, and time in MC units, which leads to a (monomer) diffusion constant of order one), and omitted prefactors of order unity. The model used in the MC simulations has a finite stretchability, while a Gaussian chain can be stretched indefinitely. However, once the scaled force $f \equiv F a / k_{B} T$ becomes significantly larger than unity, the dynamics becomes essentially independent of $F$, as a backwards step of the end point has negligible probability. Thus, instead of using $F \rightarrow \infty$, we can get set $f=1$, which leads to the boundary condition $\partial x / \partial n=-1$ at the beginning of the chain. For such a boundary condition we can easily find a stationary

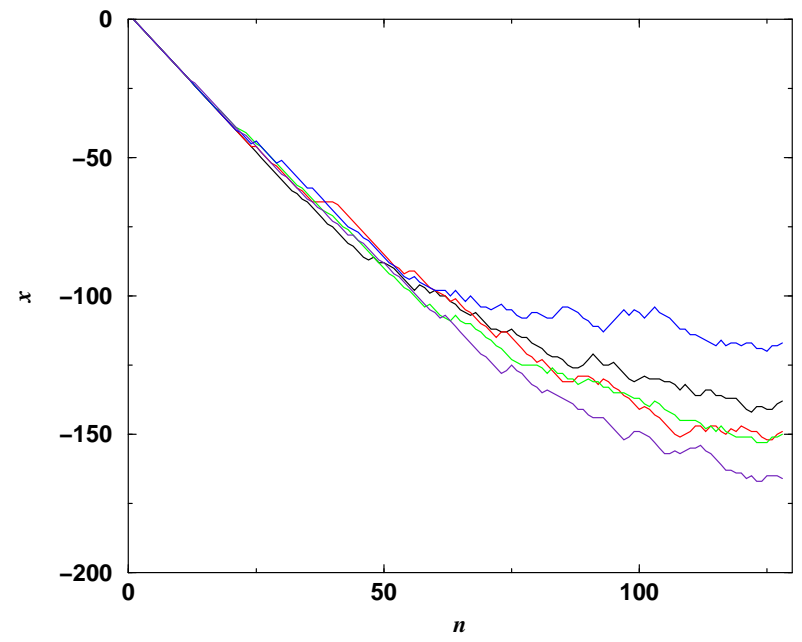

FIG. 14: Five profiles of a 1D phantom polymer of length $N=128$, with an infinite force applied to one end in steady state motion. The curves are displaced along the vertical axis such that the position of the first monomer is at $x=0$.

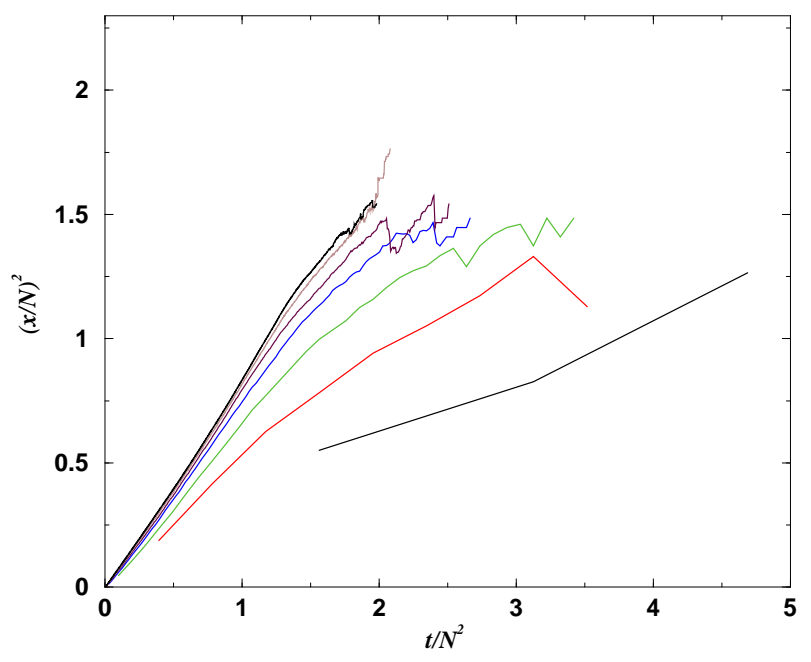

FIG. 15: Scaled coordinate $x$ of the first monomer as a function of scaled time $t$, for $N=8,16, \ldots, 512$ (right to left), in the absence of a membrane.

solution of Eq. (A1), as

$$
x(n)=v t+\frac{v}{2}(n-N)^{2},
$$

where $v=1 / N$. Simulations, of our model polymer in the absence of a membrane indeed confirm that the stationary state velocity is proportional to $1 / N$. Moreover, the actual shape of the polymer in steady state, as depicted in Fig. 14 is similar on average to Eq. A2).

The solution in Eq. A1 can also be used to understand the crossover to stationary motion, starting from a relaxed initial state. It is easy to see that the initial velocity of a point to which the force is applied is of order unity. As time progresses, this velocity decays as $t^{-1 / 2}$, 


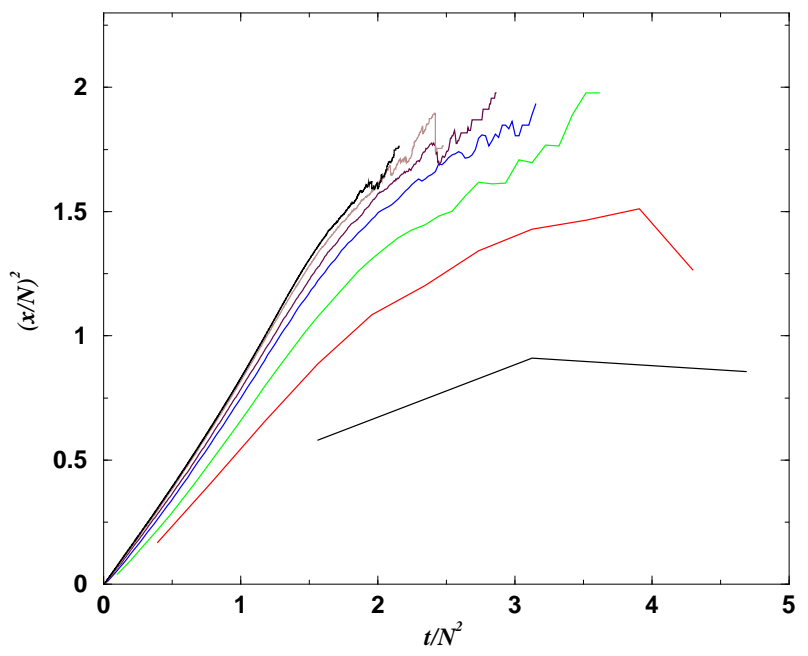

FIG. 16: Scaled coordinate $x$ of the first monomer of a translocating phantom 1D polymer, as a function of the scaled time $t$, for $N=8,16, \ldots, 512$ (right to left).

until after time $\tau \approx N^{2}$ it reaches its final (stationary) value of order $1 / N$. The monomer at the opposite end does not feel the external force in the beginning, and starts moving with velocity of order $1 / N$ after time $\tau$. We also performed simulations of unimpeded polymer motion mimicking the translocation set-up, by considering an imaginary membrane located at position $x=0$ which has no effect on the motion of monomers. The initial configuration was chosen by equilibrating the polymer on one side $(x<0)$, with the first monomer fixed to $x=0$. Then an infinite force was applied to the first monomer, and its position $x$ was tracked as a function of time, until all the monomers crossed to $x>0$. For every $N$, the results were averaged over 1000 independent runs. In these simulations the end point only needs to move a distance of order $R_{g} \sim N^{1 / 2}$ to cross to $x>0$. Moving at a steady velocity of $1 / N$, this would take a time of order $N^{3 / 2}$, which is significantly shorter than $\tau$. Thus the time $\sim N^{2}$ required for the last monomer to start feeling the force sets the time scale for a polymer with a large force applied to its first monomer, to move a distance of order of its radius of gyration. The measured $N$-dependence of the translocation time is depicted by squares in Fig. 14 There is a slight curvature in the logarithmic plot and the slope approaches $2.00 \pm 0.01$, confirming $\tau \sim N^{2}$.

The above arguments suggest that $(x / N)^{2}$ should be a linear function of $t / N^{2}$. Thus appropriately scaled plots of the motion of the first coordinates should collapse for different values of $N$. The actual picture appearing in Fig. [15] is more complicated: while we see an approach to a single curve for the largest $N$, there are very strong finite size effects for moderate values of $N$. The results for crossing the imaginary wall are surprisingly similar to those for translocation of the phantom polymer through a hole (as described in Sec. IV], as depicted in Fig. [16] (Both plots are obtained by averaging 1000 translocation processes.) The linear behavior of these curves close to the origin confirms our expectation that at short times the velocity is proportional to $t^{-1 / 2}$. However, for scaled variables around 0.5 the line has a slight curvature, which distorts the apparent scaling relations, and creates the illusion of slightly different exponents.

\section{APPENDIX B: THE TWO-DIMENSIONAL SELF-AVOIDING POLYMER MODEL}

We used a 2D lattice fluctuating bond polymer model 25] for MC simulations of a self-avoiding polymer. The monomers are placed on the sites of a square lattice, with the bonds between adjacent monomers restricted not to exceed $\sqrt{10}$ lattice constants. The excluded volume between monomers is implemented by requiring that no two monomers can approach closer than 2 lattice constants. The membrane with a hole is constructed from a row of immobile monomers arranged in a straight line, with a 3 lattice constant gap representing the hole. Such a hole is small enough to allow only a single monomer to pass through, thus enabling a unique identification of the monomer $s$ which separates the polymer into two segments on different sides of the membrane. An elementary $\mathrm{MC}$ move consists of randomly selecting a monomer and attempting to move it onto an adjacent lattice site (in a randomly selected direction). If the new position does not violate the excluded-volume or maximal bond-length restrictions, the move is performed. $N$ elementary moves form one MC time unit. The first monomer is not allowed to withdraw to the opposite side of the membrane. Since we are investigating a non-equilibrium process, the initial conditions may play an important role. We chose an initial state in which the first monomer was fixed inside the hole, and the remaining polymer was equilibrated for more than the Rouse relaxation time [11]. After such equilibration, the first monomer was released, and that moment was designated as $t=0$.
[1] B. Alberts, Molecular Biology of the Cell, Garland Publishing, New York (1994).

[2] B. Hanss, E. Leal-Pinto, L. A. Bruggeman, T. D. Copland, and P. E. Klotman, Proc. Nat. Acad. Sci. USA 95, 1921 (1998).

[3] J. Han and S. W. Turner and H. G. Craighead, Phys.
Rev. Lett. 83, 1688 (1999).

[4] S. E. Henrickson and M. Misakian and B. Robertson and J. Kasianowicz, Phys. Rev. Lett. 85, 3057 (2000).

[5] A. Meller, L. Nivon, and D. Branton, Phys. Rev. Lett. 86, 3435 (2001).

[6] J. Kasianowicz, E. Brandin, D. Branton and D. Deamer, 
Proc. Natl. Acad. Sci. USA 93, 13770 (1996); M. Akeson, D. Branton, J. Kasianowicz, E. Brandin and D. Deamer, Biophys. J. 77, 3227 (1999); A. Meller, L. Nivon, E. Brandin, J. Golovchenko, and D. Branton, Proc. Natl. Acad. Sci. USA 97, 1079 (2000).

[7] A. Baumgärtner and M. Muthukumar, J. Chem. Phys. 87, 3082 (1987).

[8] M. Muthukumar and A. Baumgärtner, Macromolecules 22, 1937 and 1941 (1989).

[9] G. W. Slater and S. Y. Wu, Phys. Rev. Lett. 75, 164 (1995).

[10] E. Arvanitidou and D. Hoagland, Phys. Rev. Lett. 67, 1464 (1991); D. A. Hoagland and M. Muthukumar, Macromolecules 25, 6696 (1992); P. Mayer, G. W. Slater, and G. Drouin, Appl. Theor. Electrophoresis 3, 147 (1993); N. A. Rotstein and T. P. Lodge, Macromolecules 25, 1316 (1992); I. Szabo, G. Bathori, F. Tombola, M. Brini, A. Coppola, and M. Zoratti, J. Biol. Chem. 272, 25275 (1997).

[11] P.-G. de Gennes, Scaling Concepts in Polymer Physics, Cornell Univ. Press, Ithaca (1979).

[12] M. Muthukumar, J. Chem. Phys. 111,10371 (1999).

[13] M. Muthukumar, Phys. Rev. Lett. 86, 3188 (2001).

[14] P. G. de Gennes, Physica A 274, 1 (1999).

[15] D. K. Lubensky and D. R. Nelson, Biophysical Journal 77, 1824 (1999).

[16] A. Baumgärtner and J. Skolnick, Phys. Rev. Lett. 74, 2142 (1995).

[17] R. Zandi, D. Reguera, J. Rudnick, W. M. Gelbart, preprint cond-mat/0301331 (2003).
[18] P. J. Park and W. Sung, J. Chem. Phys. 108, 3013 (1998).

[19] E. Slonkina, A. B. Kolomeisky, J. Chem. Phys. 118, 7112 (2003).

[20] H. C. Loebl, R. Randel, S. P. Goodwin, and C. C. Matthai, Phys. Rev. E 67, 041913 (2003).

[21] C. Y. Kong and M. Muthukumar, Electrophoresis 23, 2697 (2002).

[22] A. Meller, J. Phys.: Cond. Matt. 15, R581 (2003).

[23] M. Doi and S. F. Edwards, The Theory of Polymer Dynamics, Clarendon Press, Oxford (1986).

[24] K. Kremer and K. Binder, J. Chem. Phys. 81, 6381 (1984); G.S. Grest and K. Kremer, Phys. Rev. A 33, 3628 (1986).

[25] I. Carmesin, K. Kremer, Macromolecules 21, 2819 (1988).

[26] J. Chuang, Y. Kantor, and M. Kardar, Phys. Rev. E 65, 011802 (2001).

[27] U. Gerland, R. Bundschuh and T. Hwa, preprint cond-mat/0306126 (2003).

[28] Z. Farkas, I. Derenyi, T. Vicsek, J. Phys.: Cond. Matt. 15, S1767 (2003).

[29] N. Madras and G. Slade, Self-Avoiding Walk, Birkhäuser, Boston (1999).

[30] S.-S. Chern, A. E. Cádenas, and R. D. Coalson, J. Chem. Phys. 115, 7772 (2001).

[31] R. Metzler and J. Klafter, in Adv. Chem. Phys. 116, p. 223, ed. by I. Prigogine and S. A. Rice, Wiley (2001), and references therein. 